\title{
Analysis of a Flexible Transit Network in a Radial Street Pattern
}

\author{
Hongguo Shi ${ }^{1,2}$ and Mingyao Gao $\mathbb{i}^{1}$ \\ ${ }^{1}$ School of Transportation and Logistics, Southwest Jiaotong University, Chengdu 611756, China \\ ${ }^{2}$ School of Traffic and Logistics Engineering, Xinjiang Agricultural University, Urumqi 830052, China \\ Correspondence should be addressed to Mingyao Gao; gmy@my.swjtu.edu.cn
}

Received 16 March 2019; Revised 13 October 2019; Accepted 2 November 2019; Published 17 January 2020

Academic Editor: Abdelaziz Bensrhair

Copyright (c) 2020 Hongguo Shi and Mingyao Gao. This is an open access article distributed under the Creative Commons Attribution License, which permits unrestricted use, distribution, and reproduction in any medium, provided the original work is properly cited.

\begin{abstract}
Traditional transit systems are usually composed of fixed routes and stops, which are suitable in densely populated areas. This paper presents a reformulation of the flexible transit model developed by Nourbakhsh and Ouyang (2012) to adapt it to many low demand cities in the world, especially those characterized by radial street patterns. Unlike traditional ones, buses of the proposed transit network are allowed to traverse in a predetermined service area and their precise trajectories hinge on the exact locations of passengers. To identify the optimal topology structure of the flexible transit system, continuous approximation approaches are developed to explore the optimal value of design parameters of the whole system, defining the optimal network layout through minimizing its objective function. To exhibit its advantages, numerical experiments are conducted to compare the flexible transit system with its two variants. The results show that the flexible transit system proposed in this paper outperforms the other two variants. The higher the access cost is, the more it would tilt towards the flexible transit system with a significant margin. Besides, the flexible transit system in a radial pattern competes more effectively than that in a grid structure. This is encouraging because the proposed transit system can be applied in a number of real-world cases.
\end{abstract}

\section{Introduction}

As a mode of collective transportation, public transit has been flourishing over the past few decades due to the severely congested traffic and polluted air condition in metropolitan cities. Traditional transit, functioning as a high-capacity vehicle, is favorable in densely populated areas. However, in well-developed countries, due to low demand density, operating the transit system exactly according to the traditional mode is indeed unwise, surely leading to waste of resources. To help the transit system stay above water, specific efforts of planning, designing, and transforming transit system are desirable. Indispensable in this effort is to transform the way by which the transit systems are designed and operated $[1,2]$.

The overwhelming majority of transit design research focus on choosing certain fixed routes and frequencies such that the whole system cost is minimized. To attract more passengers to transit, an efficient and effective way is to inject new vitality to the operation of the transit service. To this end, an improvement in transit network topology and operations management is the main element. This paper is concerned with the optimal design of a novel flexible-route transit system that couples the hybrid structure and the flexibility of the demand responsive service (DRS). Some research needs to be conducted before a flexible transit system envisioned above can be configured with more details.

In this paper, a novel flexible transit system designed is adapted to cities where the travel demand density is relatively low. To the best of our knowledge, traditional transit system is usually composed of buses operating along fixed routes and stopping at fixed bus stops. However, such an operating mode tends to be more suitable for high travel demand density areas, such as many cities in Asian countries. However, in some cities of Europe, America and many other well-developed countries, these traditional transit systems do not make good use of resources and will no doubt perform better with some adjustment. Otherwise, the optimal spacing between transit lines and stops are likely to be rather large, which will definitely render passengers in an inconvenient and uncomfortable situation.

In the world, different cities have different street patterns, some form a grid structure, such as Beijing, Chicago and so 
on, while others may take on a radial pattern, composed of radial axes and concentric rings, such as Moscow, Paris, Madrid, Amsterdam, Milan, Berlin, and Palma [3]. As we know, the flexible transit system defined by Nourbakhsh and Ouyang [4] can be adapted to cities with grid street patterns nicely, but not to a radial one directly. The differences between the topology patterns necessitate the development of a different analytical model from the previously developed ones to determine the optimal design in a radial structure, which is what this paper intends to focus on. To this end, a continuous approximation approach is employed to establish the optimal design model, which is expressed as a mixed integer problem and then solved by an optimizer.

The highlights of the proposed model are as follows:

(i) A flexible transit system is initiated with no fixed routes and stops with a radial street pattern, which seems to have garnered less attention in the current literature.

(ii) The flexible transit system bears some characteristics of demand responsive transit (DRT). However, it also has its own unique characteristics. Each vehicle in the flexible transit system operates in its own predetermined "bus tube" rather than "wandering" in the whole service area aimlessly.

(iii) The flexible transit system shows great potential and benefits under certain demand levels. It has enough flexibility to adapt to the changes of travel demand density. For example, with the travel demand density exceeding a certain threshold, the transit system can run in line with the circular routes concentric to the center and the radial routes passing through the center, picking up, and dropping off passengers at designated positions rather than the exact position of passengers.

(iv) To bypass the complexity in establishing and solving the problem, continuous approximation models are developed to obtain the optimal transit network topology.

The remainder of this paper is structured as follows: in Section 2, we give a literature review on related topics involving transit network design and continuous approximation approaches. In Section 3, the structure of the flexible transit system is initiated and described, along with the formulation of the optimal design problem. In Section 4, numerical experiments and comparisons with other two comparable transit systems are conducted. Sensitivity analysis is then conducted in Section 5. Eventually, we summarize the results, point out the limitations and put forward future research in Section 6.

\section{Literature Review}

Relevant research on the transit network design problem (TNDP) is furnished in the following section. Transit network design problems have been intensively explored for quite a long time since 1960s. Various models have been developed. Among them, the majority deal with the problem by choosing a system of fixed routes and corresponding frequencies to serve a certain travel demand (see [5]). The problems are always nonlinear, nonconvex. Thus they are solved by heuristics (see e.g., [5-13]). The current models can be classified based on route structure, which includes (1) linear corridors that connect residential areas with business districts (CBD) [14-16]; (2) parallel corridors with perpendicular feeder lines [17-22]; (3) rectangular grid [23-25]; (4) radial network with $\mathrm{CBD}$ at the center $[3,26-30]$; (5) hub-and-spoke networks where the hub is a large street [31]. As regards the optimal design models, these models can be split into two categories in general. In the first category, input parameters and decision variables take values that are discrete. Travel demands are expressed as origin-destination (OD) matrix and the locations available for transit stops and routes are constrained by detailed street topology. Models of this type can obtain exact design solutions. Yet the realism embedded in these discrete models adds great complexity. It may sometimes only be tackled using mathematical approaches to obtain optimal results in a small network, however, with the enlarged size of the network, the problem tends to be quite difficult and cannot be solved using traditional methods. Thus heuristics are developed to solve it. Nevertheless, the results that are obtained donot guarantee an optimal or near-optimal design, which indeed limits its application in real cases. See e.g., grid structure [32], radial structure [27], radial/circular structure, hub-and-spoke structure [31]. Another class of models is continuous approximation models, which use "continuously changing" variables as their inputs. It omits most of the real world complex details existing in discrete models in favor of simplicity and compact. Given their advantages, they are widely used in large size applications. In the CAs, only a small number of continuous functions are established; e.g., travel demand is expressed as a density function, while lines and stations are specified in terms of the spacing between them [33-35].

Unlike discrete models, the continuous approximation models unveil the relations between inputs and outputs, which can instruct and support real world applications greatly [14, 20, 24, 28]. Tracing back to the beginning of transit network design study, [23] did the pioneering work to explore a grid transit system, followed by a pile of works $[5,35]$. Thompson [36] made a simple comparison between four different structures: radial, ubiquitous, grid, and timed transfer. It was found that a transfer-based system offers a better service for peripheral trips than the traditional radial scheme, and, at the same time, it is cheaper than a ubiquitous scheme. Newell [31] argued that a central area, where the major part of activities is concentrated, is generally found in cities due to economies of scale. Therefore, he maintained the idea that a radial system concentrated around a central corridor would be the best strategy. Jara-Díaz and Gschwender [37] studied the same problem in a simple spatial system, only composed of five nodes that form a cross. Two different strategies to serve the demand are compared: direct trips by means of lines that connect the different pairs O/D demanded, or by only two perpendicular corridors with a transfer point at their intersection. Their conclusion was that working with two corridors became more convenient with the demand disparity, and it is conditioned 
by the level of demand and unit user and agency costs. Thompson and Matoff [38] and Brown and Thompson [39] justified the change of transit system approach to transfer-based by comparing the supply, ridership, efficiency, and effectiveness of different American metropolitan areas. They concluded that those that had implemented a transfer-based network had a better performance on most measures, and had not lost productivity. Mees [40] made an exhaustive comparison about urban form and transit service between two cities, Melbourne and Toronto. The main conclusion was that the main reason why ridership in Toronto was higher than Melbourne was not related to the characteristics of their urban form, which were similar, but rather to the transit service orientation and its consequences. Toronto presented a grid transit network structure following its street pattern. Other comparisons between Boston and Toronto [41] or Broward (Florida) and Tarrant (Texas), made by Brown and Thompson [42], gave similar conclusions. There was also a similar proposal for Barcelona that is currently being implemented [34].

Recently, Daganzo [33] examined a hybrid transit system that features both the ring and radial lines in the central area and only radial lines in the city's periphery band. Estrada et al. [34] further extended Daganzo's work to a rectangular area. Nourbakhsh and Ouyang [4] designed a novel flexible transit system with no fixed transit routes. Each bus is allowed to traverse in a designated area called "bus tube" to pick up and drop off passengers. They found out that such a flexible transit system is advantageous over other transit systems when the demand density is low to moderate. Sivakumaran et al. (2014) used CAs both to explore the influence of access mode and TODs on the choice of transit technology. They found that metro and rapid transit can become more competitive if accessed by fast-moving feeders. Yet, the influence of more favorable land-use patterns tends to be negligible. Badia et al. [3] rebuilt the hybrid transit first put forward by Daganzo [33] to adapt it to cities with radial street patterns. The core of his work is to introduce stops with single coverage to improve the accessibility, striking a balance between agency cost and spatial accessibility. Such a network structure nicely inherits the advantages of both a hub-and-spoke structure (e.g., low infrastructure investment) and a grid structure (e.g., low travel time). Chen et al. [30] compared the ring-radial and grid street networks using CA optimization models. Both the models take on a hybrid fashion: intersecting routes in the center and only radial lines in the periphery. The results showed that the ring-radial networks perform better than the grid patterns. Saidi et al. [35] explored the long-term planning for ring-radial urban rail transit. In their work, the optimal number of radial lines is first examined and then the passenger route choice is analyzed according to the total travel cost rather than the shortest distance, which distinguishes their work from others'. It is found that the most important factors influencing the ring line alignment are OD patterns and the current radial network configuration. Chen and Nie $[1,2]$ analyzed two variants of an integrated e-hailing transit system: a zone-based transit one and a line-based one. They found that the line-based one competes more efficiently than the zone-based one. Further, in 2018, they studied the optimal design of demand adaptive paired-line hybrid transit in a radial route structure. Two variants are considered differentiated by the relative position of the adaptive transit lines and the fixed transit line. The results verified that the radial type outperforms the grid one. Fan et al. [43] developed continuum models to investigate the bimodel transit system. In their study, trips are grouped into five types. Then a two-level optimization problem is formulated to solve the intersecting network. The results suggested that it is wise to design the local and express lines simultaneously.

From the above review, we can find that there is a gap in the current literature on studying whether and when to establish a flexible transit system in a radial pattern and, if it is feasible, how to obtain the optimal design. In light of this, we now use CAs to address the literature's above-cited limitations.

\section{Methodology}

This section formulates the flexible transit design problem. Section 3.1 proposes the flexible transit system with a radial route structure in details. The significant metrics are calculated in Section 3.2. The optimal transit network design problem is formulated in Section 3.3.

For clarity and compactness, all the assumptions involved are listed below:

(1) The city is considered as an ideal circle of radius $R$, and its street layout shows a ring-and-radial pattern;

(2) Origins and destinations of trips are uniformly and independently distributed in the whole service area;

(3) For simplicity and comparison purposes, the spacing of ring routes is uniform;

(4) The service is provided by identical vehicles in the following operating parameters;

(5) Passengers send their demands to the flexible route vehicle dispatch center before the vehicle departs;

(6) Passengers choose the routes with the shortest distance;

(7) Passengers will not transfer to other transportation modes due to the decrease of service level. That is, the demand density is given in advance and unchanged;

(8) Each bus serves its own "bus tube" and cannot cross the lines, which means the service area of buses has been determined at the very start;

(9) No congestion effect is considered in this paper as the background of this paper is low demand density cities.

\subsection{Proposed Flexible Transit System with a Radial Route}

Structure. Consider a circular region of radius $R$ with a radial street pattern. The travel demand density is $\lambda$ passenger trips per hour per unit area. We assume that the origins and destinations of trips are uniformly and independently distributed in the whole service area. The flexible transit network is composed of nonfixed transit routes. As is illustrated in Figure 1, two types of routes are included, radial routes (boundary-centerboundary) and circular routes (concentric to the center). 


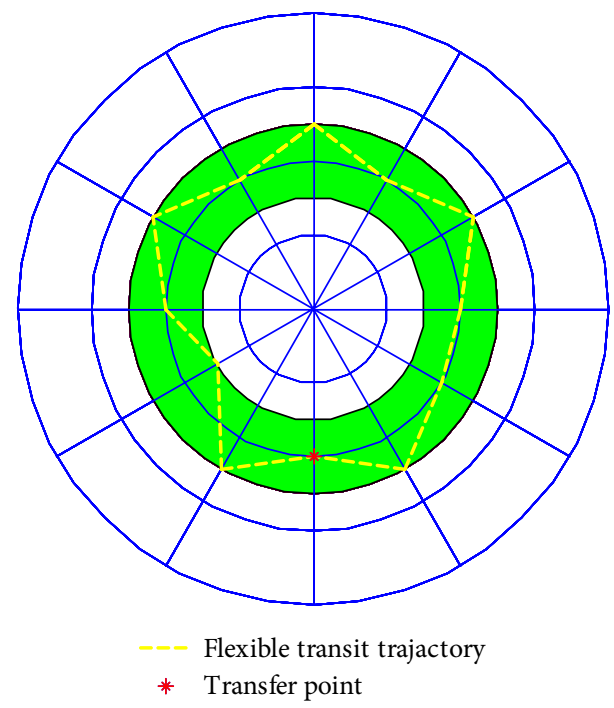

FIgURE 1: Proposed flexible transit system in a radial pattern.

Let $N_{r}$ denote the number of radial transit routes which travel from one side of the area through the center to the other side of the area. Thus, the angle between two adjacent radial routes $\theta_{r}$ is $\pi / N_{r}$. Let $N_{c}$ denote the number of circular transit routes. In a similar way, the route spacing $S_{c}$ of circular transit routes is $R / N_{c}$. The headway of transit routes is $H$. We further assume that the service is provided by identical vehicles in the following operating parameters: the cruising speed considering traffic signal and pedestrian interference, $v(\mathrm{~km} / \mathrm{h})$; the stopping time per stop due to deceleration and acceleration, $\tau_{1}$ (s/stop); the time to pick up and drop off a passenger, $\tau_{2}$ (s/pax). Thus the time per passenger stop is $\tau=\tau_{1}+\tau_{2}[44]$.

The street in the service area exhibits a ring-radial fashion. For the ease of reference, we borrow the notation "tube" originating from Nourbakhsh and Ouyang [4]. In our design, the buses make lateral movements to pick up and drop off passengers while sweeping longitudinally back and forth in their own designated "tubes", as depicted by the dash line in Figure 2.

3.2. Important Metrics of Agency and User Costs. As we all know, the system cost consists of two components: agency costs and user costs. These will be estimated for the so-called average-case user during his or her one-way trip.

The details of the formulation of costs are furnished in this subsection and the Appendix. For convenience, we list the notations involved in this paper in Table 1.

The transit network configuration is defined by three decision variables. Two of them are spatial variables that determine its topology. The third variable is the headway of service in the whole service area.

3.2.1. Agency Cost. To derive the agency cost, we first need to identify the agency cost components. Due to the fact that flexible transit routes have no fixed infrastructure, there is no fixed cost. Thus the agency cost includes two components that depend on: the expected total vehicle distance traveled per hour of operation, $Q$ and the expected total fleet size of operation, $M$.

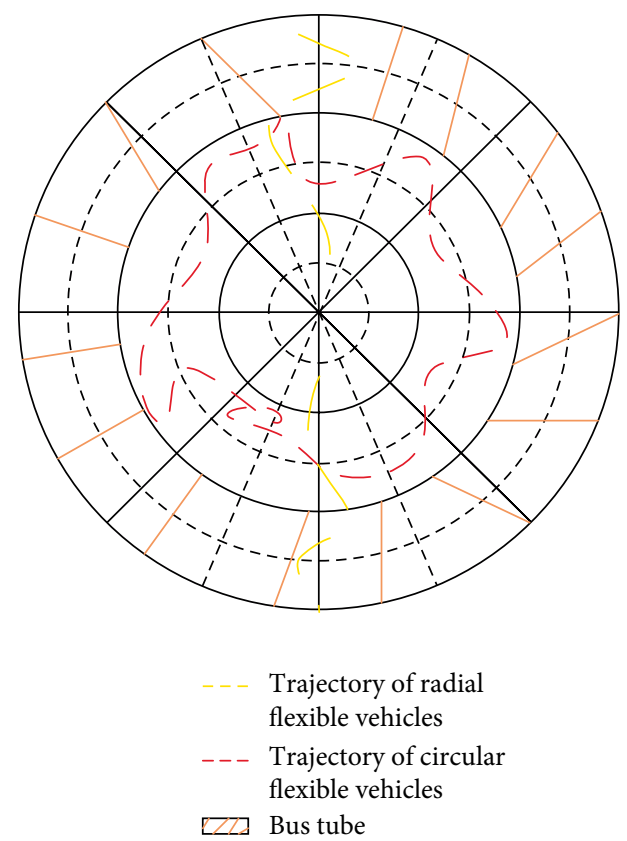

Figure 2: Trajectory of the flexible vehicle.

TABLE 1: Important notations and default values.

\begin{tabular}{|c|c|c|}
\hline Parameter & Value & Definition \\
\hline$\mu(\$ / \mathrm{h})$ & 20 & Value of time \\
\hline$\tau_{1}(s)$ & 12 & $\begin{array}{l}\text { Time cost per stop due to } \\
\text { deceleration and acceleration }\end{array}$ \\
\hline$\tau_{2}(s)$ & 13 & $\begin{array}{l}\text { Additional pick-up and drop-off } \\
\text { time required per passenger }\end{array}$ \\
\hline$v(\mathrm{~km} / \mathrm{h})$ & 25 & Vehicle’s cruising speed \\
\hline$v_{w}(\mathrm{~km} / \mathrm{h})$ & 5 & Walking speed \\
\hline$\delta(\mathrm{km})$ & 0.03 & $\begin{array}{c}\text { Transfer penalty expressed in } \\
\text { terms of the equivalent distance } \\
\text { walked }\end{array}$ \\
\hline$\$_{Q}(\$ / \mathrm{veh} \cdot \mathrm{km})$ & 2 & Operation cost per vehicle distance \\
\hline$\$_{M}(\$ / v e h \cdot h)$ & 40 & Operating cost per vehicle hour \\
\hline
\end{tabular}

(i) Vehicle distance: $Q=\left(2 \pi R N_{c} / H\right)+\left(2 \lambda \pi R^{3} / 3 N_{c}\right)$ $+\left(R N_{r} / H N_{c}\right)+\left(4 R N_{r} / H\right)+2 \lambda \pi R^{2}+\left(5 \pi R N_{c} / 12 N_{r}\right)$.

(ii) Fleet size: $M=\left(Q_{r} / v_{r}^{\prime}\right)+2 \tau \lambda \pi R^{2}+\left(Q_{r} / v_{c}^{\prime}\right)+2 \tau \lambda \pi R^{2}$.

3.2.2. User Cost. The user costs relate with the route choice of passengers. Here we adopt the assumptions to simplify the optimal design problem: (1) passengers send their demands to the flexible route vehicle dispatch center before the vehicle departs; (2) passengers choose the routes with the shortest distance.

For user costs, we consider three parts of costs: waiting time $(W)$, in-vehicle travel time $(T)$ and the transfer penalty $(P)$ for each transfer between two different transit services. Note that the transfer penalty here talked about is the extra discomfort imparted on passengers by the transfer itself rather than the time needed to transfer from one route to another. 


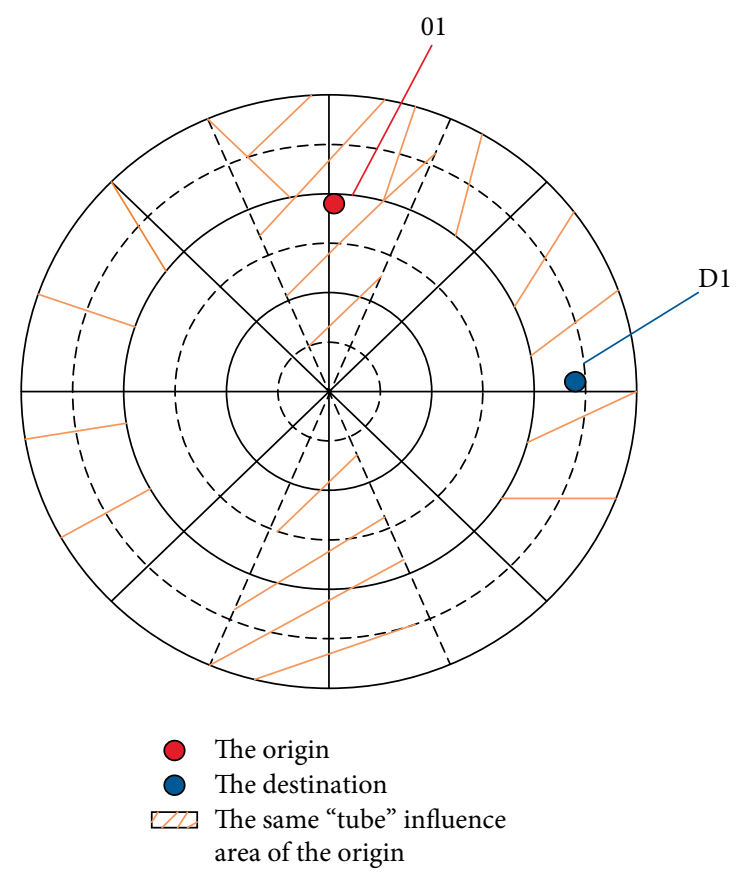

FIGURE 3: Illustration of the origin and destination of a trip.

We now first turn our attention to an important user metric: the expected number of transfers $e_{T}$ for an average user of the flexible transit services. The probability of requiring no transfer is equivalent to the probability that the origin and destination of a trip are within the same "tube" influence area (e.g., see the trip with $O$ and $D$ labeled 1 in Figure 3).

According to the number of transfers, three types of trips can be distinguished: (1) the destination is in the same radial or circular line influence area as the origin; (2) the origin and destination are not in the same line influence area and the angular distance between them is less than 2; and (3) the origin and destination are not in the same line influence area and the angular distance between them is larger than 2. Passengers in transit system are assumed to always take the shortest path. For Type 1 trips, no transfer is needed. For Type 2 trips, passengers will use both the radial line and the circular line, one transfer occurs at the intersection between the circular line and the radial line. For Type 3 trips, passengers will transfer once at the center. Hence, both Type 2 and Type 3 trips require one transfer.

3.3. Formulation of the Optimal Design Problem. The purpose of the flexible transit network design problem is to find the optimal combination of the design variables, namely, $N_{r}$, $N_{c}$, and $H$, such that the cost of the whole transit system is minimized.

As is customary, we transform the cost components into equivalent travel time. According to Daganzo [33], we suppose that $\$_{Q}$ is the cost per vehicle distance, $\$_{M}$ is the cost per vehicle hour and $\mu$ is the time value of passengers, thus $\pi_{Q}=\left(\$_{Q} / \lambda \pi R^{2} \mu\right)$ and $\pi_{M}=\left(\$_{M} / \lambda \pi R^{2} \mu\right)$ can transform the costs into equivalent travel time per passenger. Further we denote $\delta$ as the metric of discomfort of transfers imparted on passengers. Thus the equivalent travel time of transfers can be derived as $\left(\delta / v_{W}\right) e_{T}$. All together, the transit network optimization problem can be expressed as: $\min z\left(N_{r}, N_{c}, H\right)=\pi_{Q} Q+\pi_{M} M+W+T+\left(\delta / v_{W}\right) e_{T}$, where the three decision variables are embedded in the above formulation. The identification of the most competitive transit network should satisfy a proper trade-off between agency cost and user cost, between access time and in-vehicle travel time and so on.

\section{Numerical Experiments}

In the previous section, we have formulated the optimal flexible transit network design problem. As regards the approach to solve the proposed model, according to Chen and Nie $[1,2]$, Matlab's built-in generic algorithm (GA) (with default parameters on population/crossover/mutation) obtains satisfactory solutions. The constraints of the parameters used in Matlab's built-in function "ga" are the same as in the above formulation.

In this section, we compare the radial flexible transit network system with the grid one [4].The formula involved are listed in Table 2.

Solved by Matlab, the optimal decision variables and cost components are listed in Table 3.

Observed from Table 3, as expected, when the demand density increases, the average user cost reduces considerably, and the average agency cost drops relatively slightly, albeit the transfer cost remains almost intact. A plausible explanation is that with the increasing demand density, the number of transit routes rises. Thus the spacing between routes and stops is narrowed. For one thing, smaller spacing requires shorter detour distance in vehicles, which causes shorter travel distance for passengers. For another thing, passengers need to transfer a bit more, leading to the transfer cost increasing slightly.

Further, two batteries of comparisons are conducted to analyze the performance of three transit systems. Figure 4 plots the minimized system cost per passenger of the flexible transit network versus travel demand levels: (1) a small radial city of radius $R=(10 / \sqrt{\pi})$; (2) a medium-scale radial city of radius $R=(20 / \sqrt{\pi})$. We choose $(D / \sqrt{\pi}) D$ denotes the side of a square city [33] as the radius of the service area because this ensures the same area as that of the grid one.

For the fixed route transit system, the total system cost includes costs related to infrastructure establishment, vehicle distance traveled, vehicle operation, passengers' access and egress time, waiting time, in-vehicle travel time and transfer penalty. For comparison, we neglect the infrastructure cost. One may quibble over the assumption if it actually gives undue priority to the fixed route transit system, however, this assumption indeed favors the fixed route transit system and sets a high bar for success to the flexible transit system proposed in this paper.

Reading from Figure 4, it is easy to find that the flexible transit system outperforms the fixed route one with a significant margin in the low demand density. This is intuitive, when the passenger demand is low, it is unnecessary to traverse along the fixed route and stop at each fixed transit stop since no passenger may be there for the service. To exhibit the 


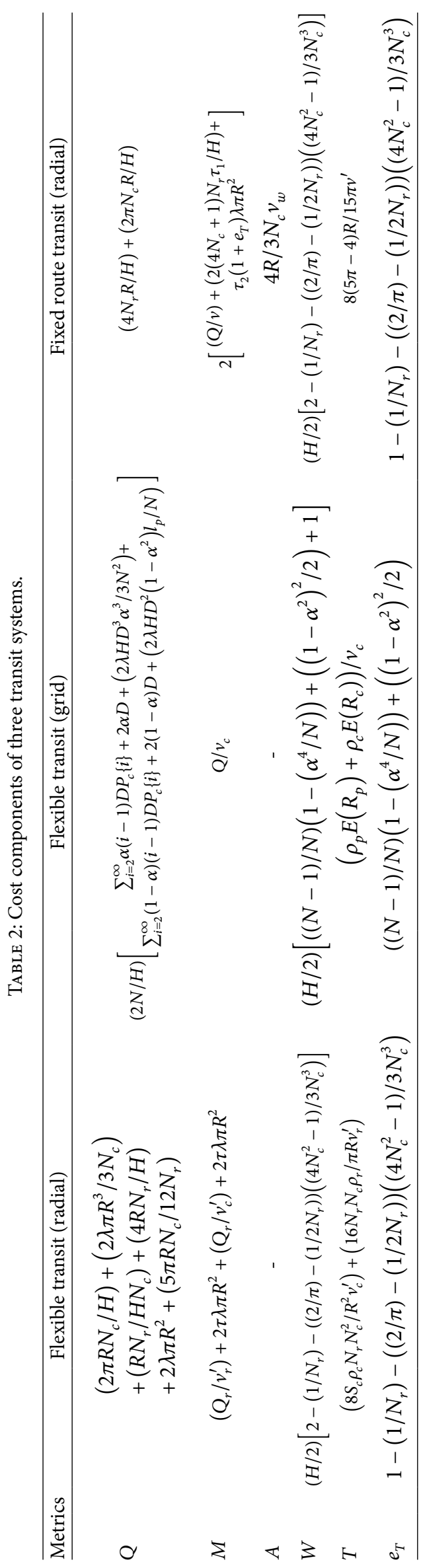


TABLE 3: Optimal decision variables and cost components.

\begin{tabular}{lcccccccccccc}
\hline$\lambda$ & $N_{r}$ & $N_{c}$ & $H(\mathrm{~h})$ & $v_{c}$ & $\$_{Q} Q$ & $\$_{M} M$ & $\begin{array}{c}\text { Agency } \\
\text { cost }\end{array}$ & $W$ & $T$ & User cost $\delta e_{T} / v_{W}$ & $z$ \\
\hline 1 & 5 & 1 & 0.300 & 22.0 & 0.62 & 0.55 & 1.17 & 0.59 & 0.52 & 1.11 & 0.028 & 2.308 \\
2 & 7 & 2 & 0.250 & 21.9 & 0.58 & 0.53 & 1.11 & 0.51 & 0.52 & 1.03 & 0.028 & 2.168 \\
5 & 9 & 3 & 0.200 & 21.4 & 0.53 & 0.49 & 1.02 & 0.42 & 0.51 & 0.93 & 0.028 & 1.978 \\
10 & 12 & 4 & 0.120 & 21.3 & 0.46 & 0.45 & 0.91 & 0.34 & 0.50 & 0.84 & 0.027 & 1.777 \\
20 & 16 & 5 & 0.060 & 20.7 & 0.41 & 0.39 & 0.80 & 0.24 & 0.50 & 0.74 & 0.027 & 1.567 \\
50 & 22 & 6 & 0.040 & 20.3 & 0.34 & 0.36 & 0.70 & 0.12 & 0.48 & 0.60 & 0.025 & 1.325 \\
100 & 28 & 7 & 0.030 & 19.6 & 0.21 & 0.34 & 0.55 & 0.09 & 0.47 & 0.56 & 0.025 & 1.135 \\
200 & 30 & 8 & 0.020 & 19.2 & 0.19 & 0.27 & 0.46 & 0.08 & 0.46 & 0.54 & 0.024 & 1.024 \\
500 & 58 & 12 & 0.017 & 18.3 & 0.16 & 0.21 & 0.37 & 0.06 & 0.45 & 0.51 & 0.023 & 0.903 \\
\hline
\end{tabular}

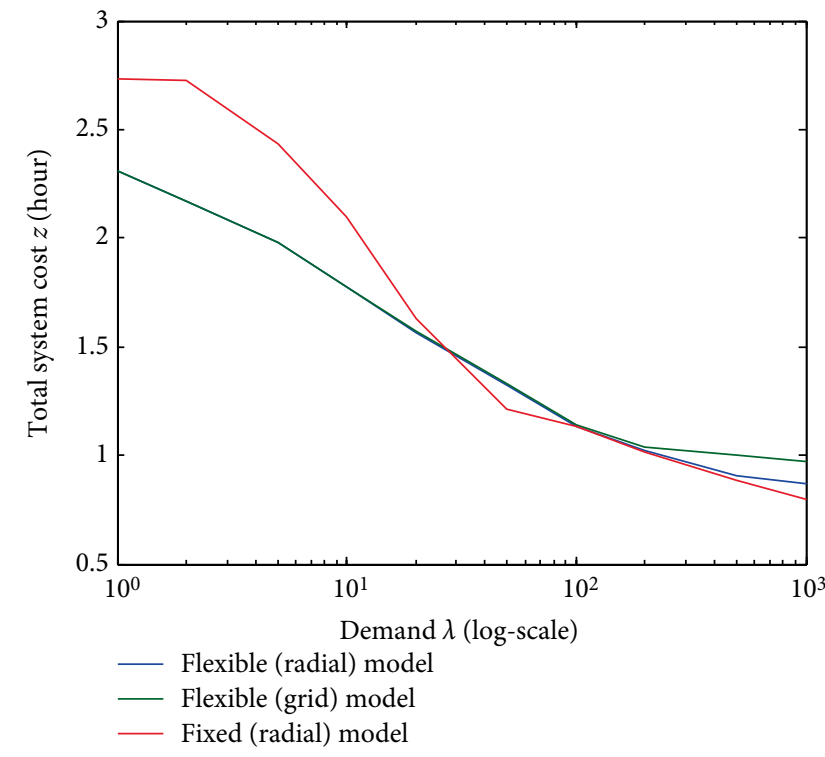

(a)

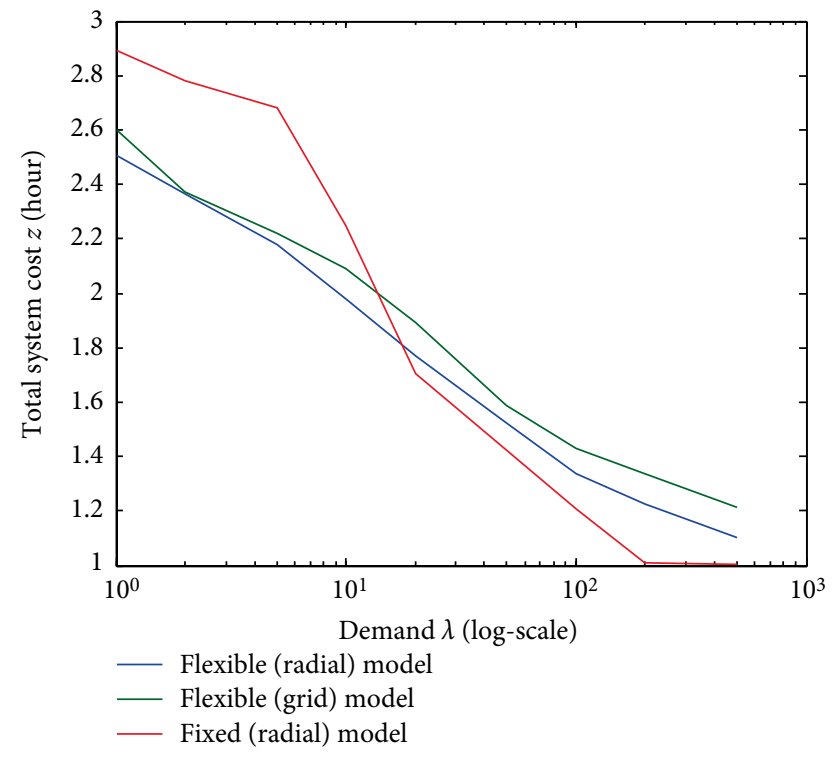

(b)

Figure 4: Minimized system cost versus demand density levels. (a) A small city $(D=10 \mathrm{~km}, R=5.64 \mathrm{~km})$. (b) A mid-size city $(D=20 \mathrm{~km}$, $R=11.28 \mathrm{~km})$.

impact of the street structure on the flexible transit design problem, we compare the flexible transit system proposed in this paper with its counterpart in a grid network [4]. In their paper, the buses traverse in certain bus "tubes" in a grid street structure. To eliminate the external factors, we set the same area of the service areas in both the radial and grid patterns. The results demonstrate that the flexible transit system in a radial structure also wins over the grid one slightly.

Figure 5 plots the optimal decision variables of the transit networks versus demand levels.

The number of both the radial routes and circular routes increases with the demand levels as we anticipated. It is striking to see that the increase of headway of the whole transit system tends to be more dramatic, compared with the other two decision variables.

Figure 6 plots the minimized agency costs and user costs against demand levels. A notable observation can be found that the agency cost is more sensitive to the travel demand density than the user cost by a large margin. When the value of $\lambda$ rises up to 100 from 1 , the agency cost drops sharply about
$60 \%$ while the user cost drops slightly. The reason may be that when the travel demand density increases, the agency may benefit from economies of demand density, however, the user costs canot decrease endlessly beyond a critical level since the overcome distance exists. That is, no matter which level the travel demand reaches, a minimum value of travel distance must be overcome as the origin and the destination have a spatial distance which cannot be diminished.

Here comes more detailed analysis of different cost components. Figure 7 demonstrates that passengers suffer more in-vehicle travel time relative to the total travel time, which is exactly more obvious in the grid flexible transit system. This is not strange because the real transit routes between the origins and the destinations of trips in a grid transit system are more roundabout than those in radial ones. This may be attributed to the manner that the radial network furnishes more direct routes to passengers compared with grid networks.

Analyzing the figures above in depth, it is obvious that apart from remarkable advantages, such as more convenient 


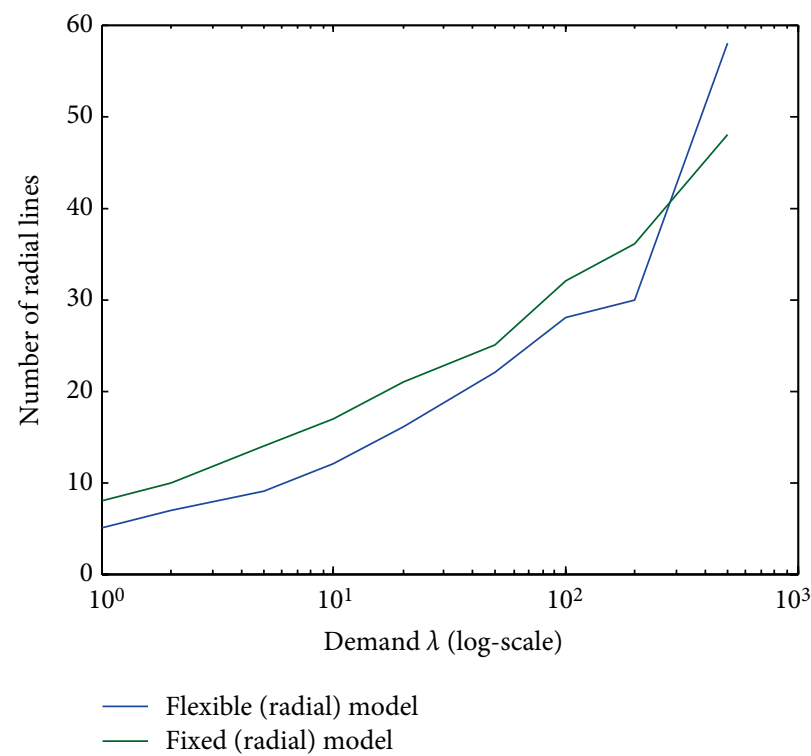

(a)

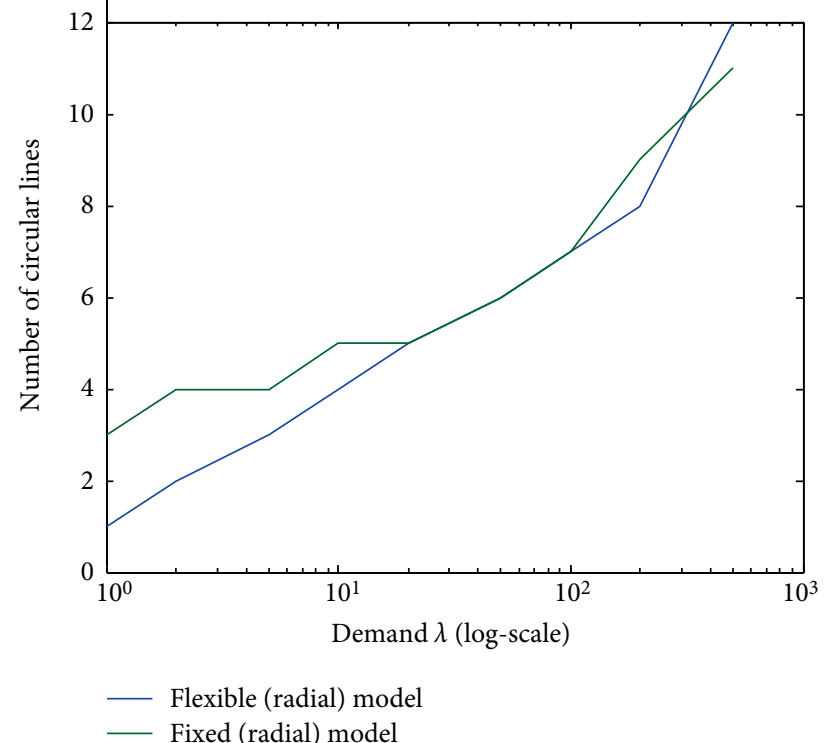

(b)

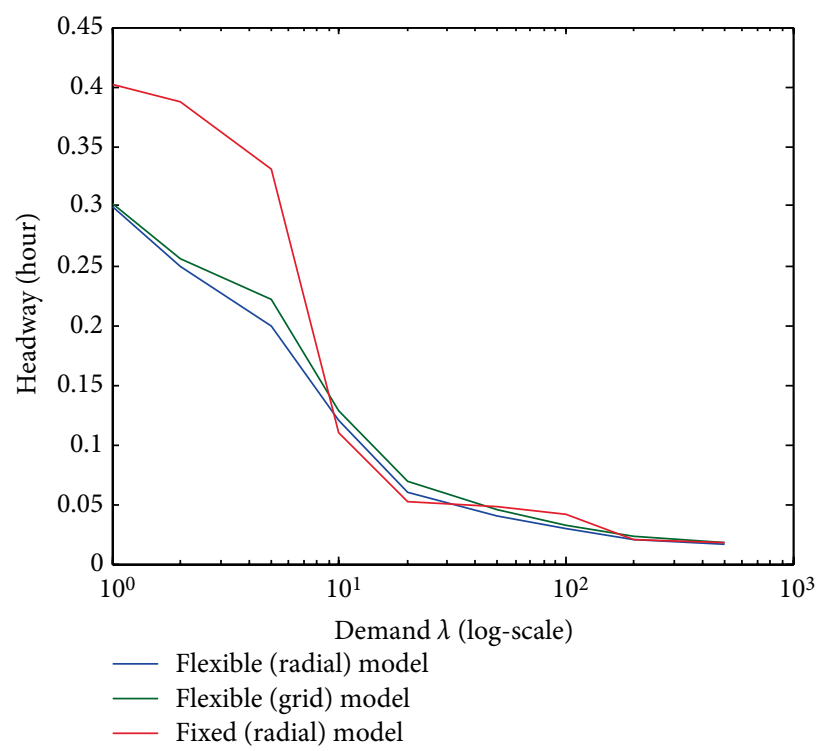

(c)

FIGURE 5: Optimal decision variables of three transit systems. (a) Number of radial lines. (b) Number of circular lines. (c) Headway of three transit systems.

and resource-saving, the flexible transit system has its own defect. Unlike the fixed route transit system, the flexible transit operates in strict accordance with the realization of passengers (the realization of passengers means that the exact location of passengers), which may impose an extra detour distance on passengers especially those who get on the buses early and this can cause reduction of service quality.

Seeing from Figure 7(b), when the demand is low, the travel times of the flexible transit system is slightly longer than that of the fixed transit system. However, when the demand increases to a certain level, it incurs much more in-vehicle time than the fixed route transit system, which is the product of extra detour brought by door-to-door service.
A few reasons can explain why the flexible transit system dominates in low density areas. Firstly, it eliminates the access and egress time of passengers at fixed stops and the cost savings from the former are more than the offset by the latter. Secondly, due to the low travel demand density, the operating vehicles can choose from minibuses instead of traditional high-volume ones, which, to a great extent, cut down the agency cost as well.

Note that in all the figures, user costs drops less than the agency costs. This happens because agency costs can be reduced as much as possible by choosing large spacing and (or) headways, but user costs cannot be reduced below the time threshold to overcome the direct distance-between the origin and destination of a trip. 


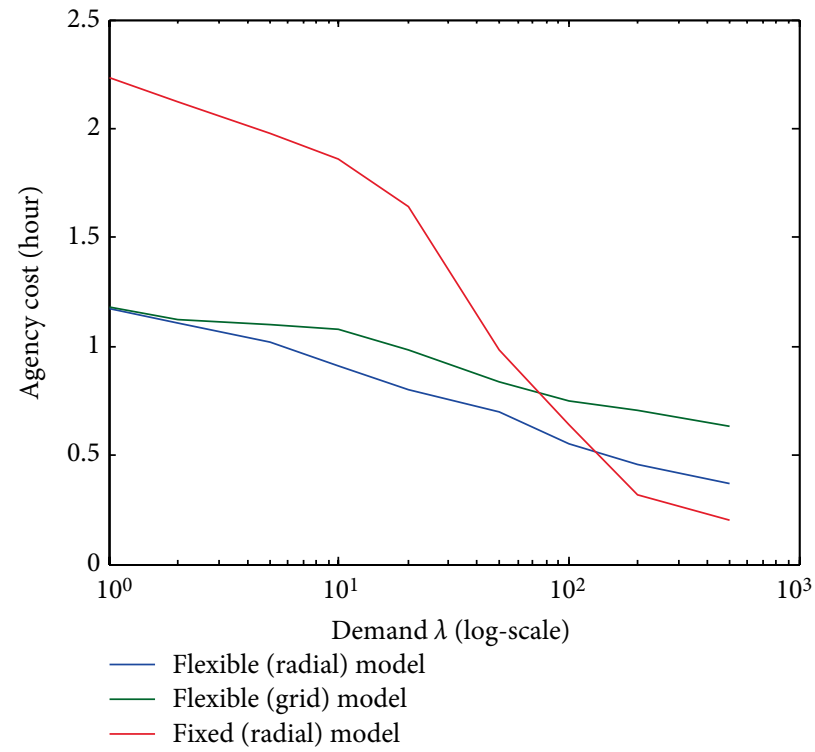

(a)

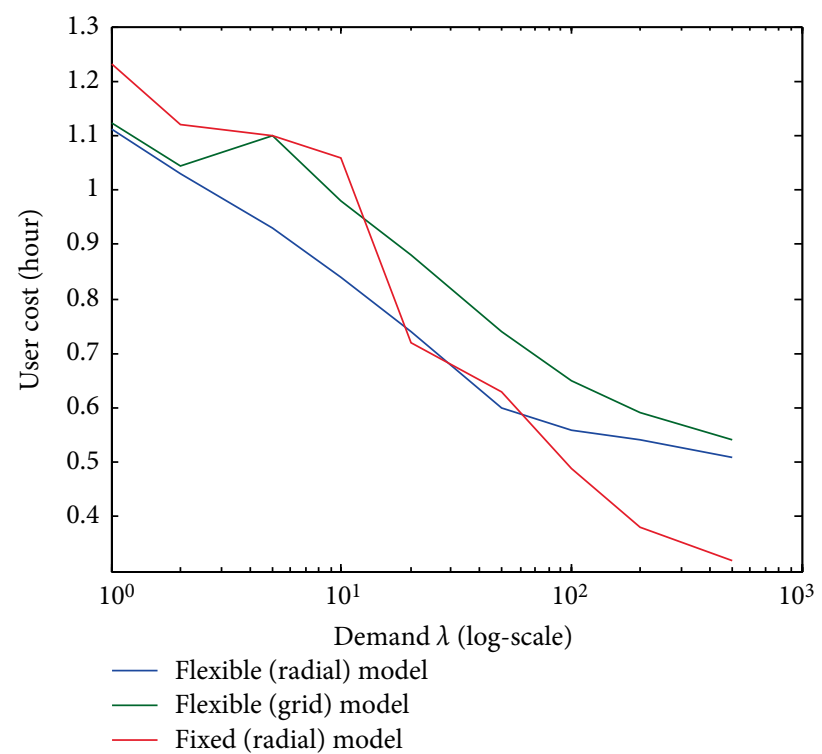

(b)

Figure 6: Agency and user cost of three transit systems. (a) Agency cost versus demand density levels. (b) User cost versus demand density levels.

\section{Sensitivity Analysis}

In this section, we examine how the input parameters of the flexible transit system influence the optimal design. A wide range of scenarios are analyzed.

To test the impact of passenger stopping time, we choose a more conservative value of $\tau=24 \mathrm{~s}$. Intuitively, the longer the passenger stopping time, the more travel time passengers will have to endure. The effect should be more obvious for the flexible transit system as each passenger need a stop for both the get-on and get-off. To this end, we conduct a sensitivity analysis to see the real cases and the results are displayed in Figure 8.

Figure 8(a) plots the minimized system cost versus the demand levels with the stop time per passenger increases from $12 \mathrm{~s}$ to $24 \mathrm{~s}$. As clearly shown in Figure 6(b), all the three total cost increases compared with values in Figure 4(a), especially the flexible transit system in a grid network.

Figure 8 (b) plots the minimized system cost versus the demand level when walking speed takes a low value. Such circumstances might occur when the weather is extremely terrible or on a dark night, which blocks passengers from accessing the transit system. Thus, the fixed route transit system is forced to be put in an unfavorable situation because all passengers are assumed to walk to the fixed transit stations without any other access approaches. The system cost of the flexible transit also increases slightly, which may result from the increased transfer time.

Figure 8 (c) plots the minimized system cost versus the demand level when the walking speed rises by $50 \%$. As can be read from Figure 8(c), all three curves follow the same trend, however, the gap between the flexible transit system and the fixed route system narrows. This is expected since the flexible transit system is designed to eliminate the access and egress time. In the condition of high walking speed, the flexible transit system will undoubtedly lose its superiority. Yet, the reduced access and egress time, to some extent, compensates for the longer in-vehicle travel time, which brings down the total system cost of the flexible transit system.

Figure $8(\mathrm{~d})$ plots the minimized system cost versus the demand level when the value of time of passengers reaches up to four times as the former one. Compared with Figure 1, the benefits of flexible transit system are dwarfed. The more costly the in-vehicle time, the more unfavorable the flexible transit system. Another observation is that the total cost of the fixed route transit does not change perceptibly as the flexible one, with discrepancies less than ten percentage.

\section{Conclusion}

As we all know, a transit system can be more efficient (less system cost) and more effective (more passengers) by eliminating the access and egress time. Efforts in this aspect usually include three approaches: (1) providing high-speed feeders as connectors; (2) transforming the land-use patterns, such as Transit Oriented Development (TOD); (3) improving the vehicle operating mode. The former two approaches have been intensively talked about in the past. The last one is what this paper intends to focus on. To this end, this paper proposes a novel flexible transit system with buses traversing in their own designated "tubes". Through analysis of all relevant agency costs and user costs, we utilize the continuous approximation approach with several variables to establish the optimal design problem as a mixed integer program, which derives a concise formulation and can be easily solved by an optimizer, such as Genetic Algorithm (GA) embedded in Matlab.

Owing to the inherent characteristics of the approach applied, the outcome obtained casts important insights into the internal relation of the input parameters and the outcome. The main findings are listed as follows: 


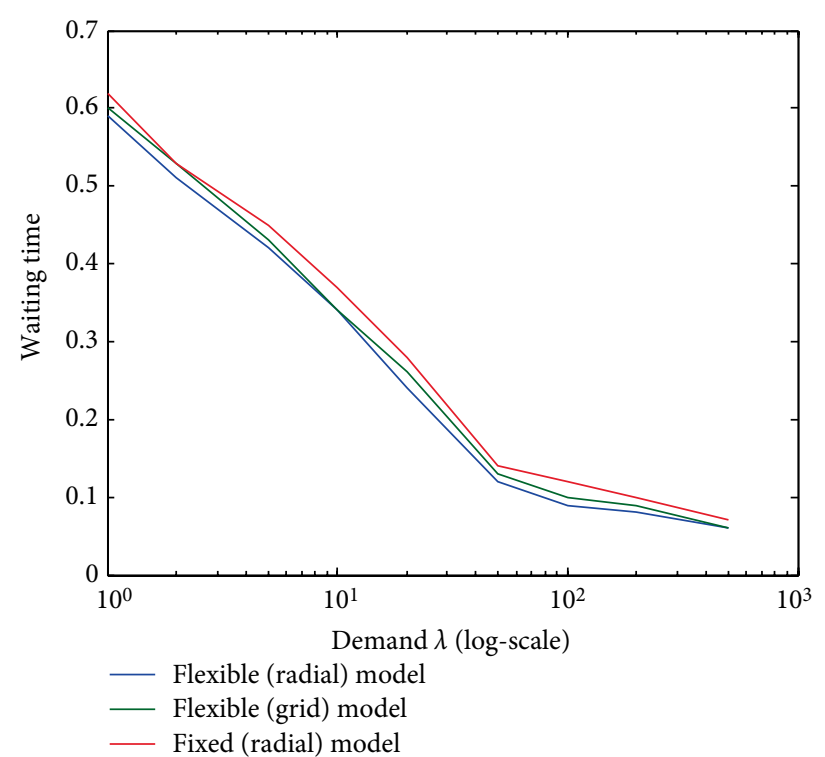

(a)

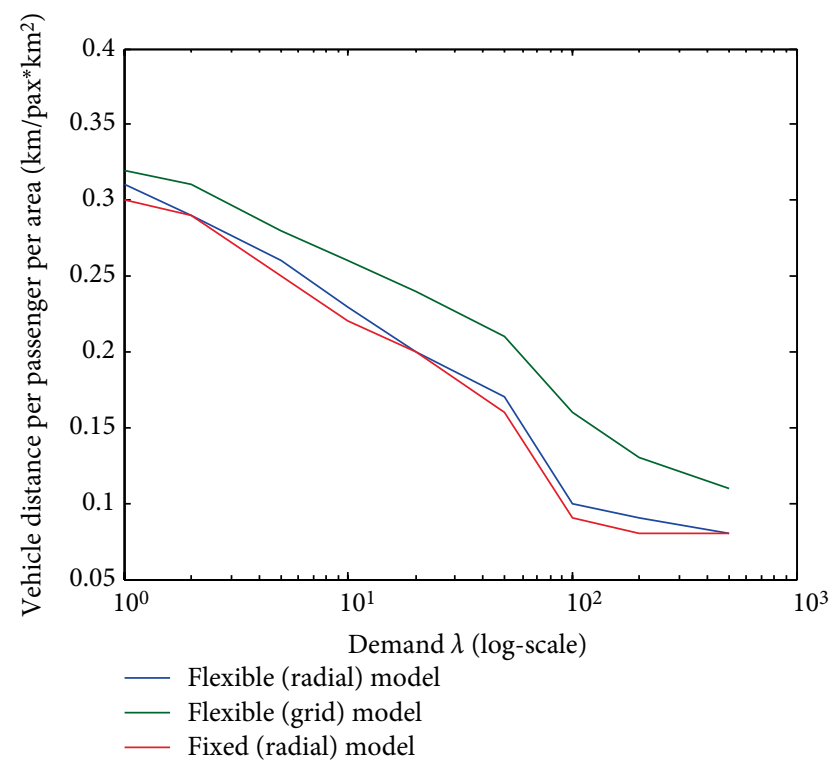

(c)

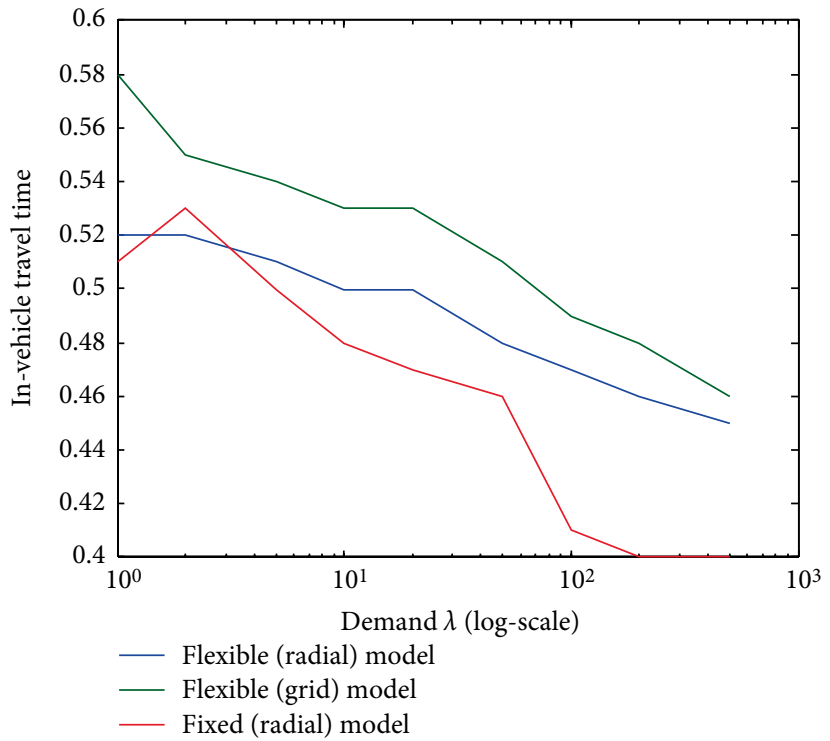

(b)

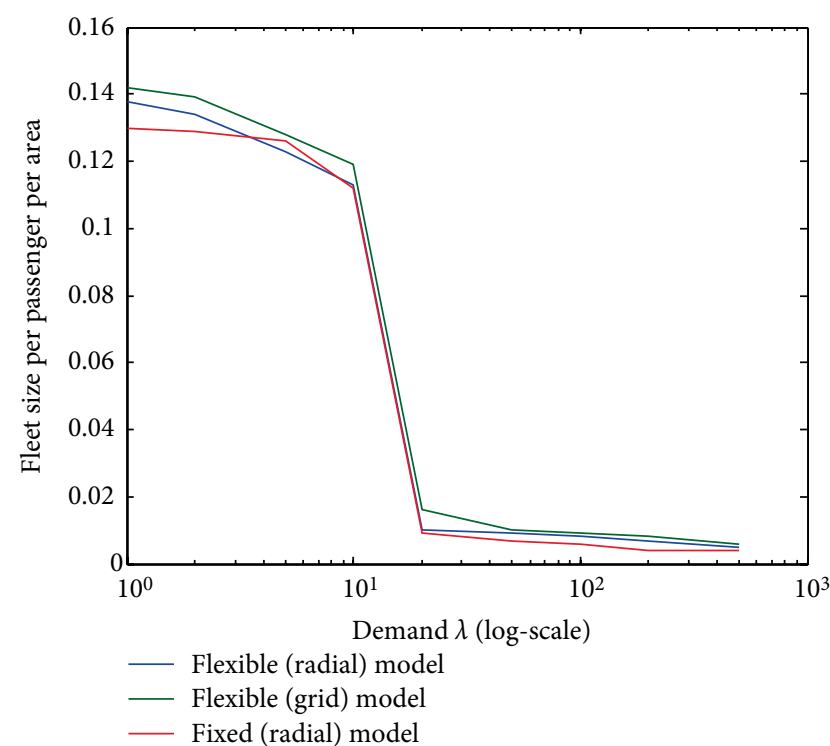

(d)

FIGURE 7: Different cost components of three transit systems. (a) Waiting time versus demand levels. (b) In-vehicle travel time versus demand levels. (c) Vehicle distance versus demand levels. (d) Fleet size versus demand levels.

(i) The comparisons between the flexible transit system proposed in the paper and its variant $[4,44]$ demonstrate that in low travel demand density areas, the flexible transit network designed in this paper is advantageous over the fixed one with a significant margin. This is inspiring because it means the design can be applied to a list of real-world instances.

(ii) Compared with fixed route transit network systems, the proposed flexible transit system is advantageous in certain aspects. Above all, it enables passengers to gain access to the transit system easily without walking a long time to the designated transit stops. What is more, it reduces agency cost significantly as well because it has no infrastructure cost. However, passengers may have to travel a bit longer in vehicles at a cost due to the vehicle's lateral movements for picking up and dropping off passengers. When the travel demand density is relatively low, the extra lateral distance is negligible and the whole system is optimal. However, when the travel demand density is moderate to high, the extra lateral distance will impose high in-vehicle travel time on passengers, which is more than offset by the elimination of other cost savings and thus renders the flexible transit system at a great disadvantage.

(iii) When the demand is high, the fixed route transit is more efficient. Thus the flexible transit system can be converted into the traditional ones via operating along fixed routes and stopping at designated transit stops, which can adapt to the changes of travel demand density easily and conveniently. 


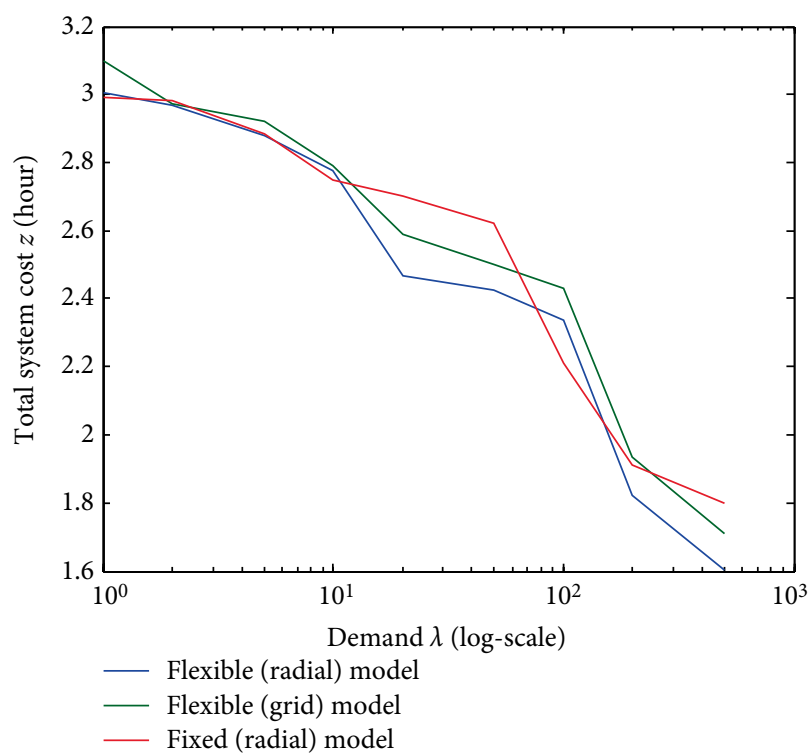

(a)

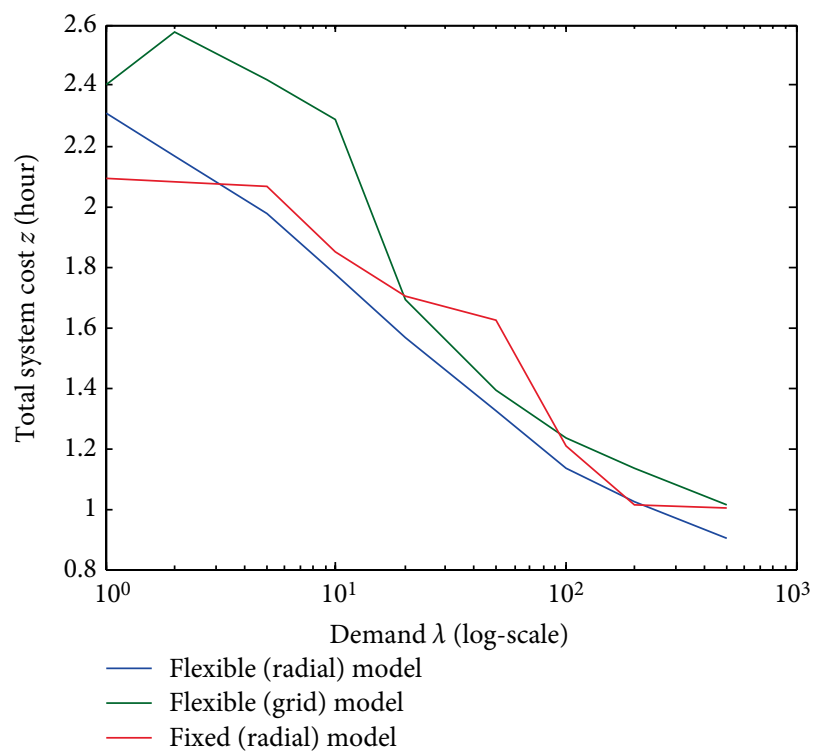

(c)

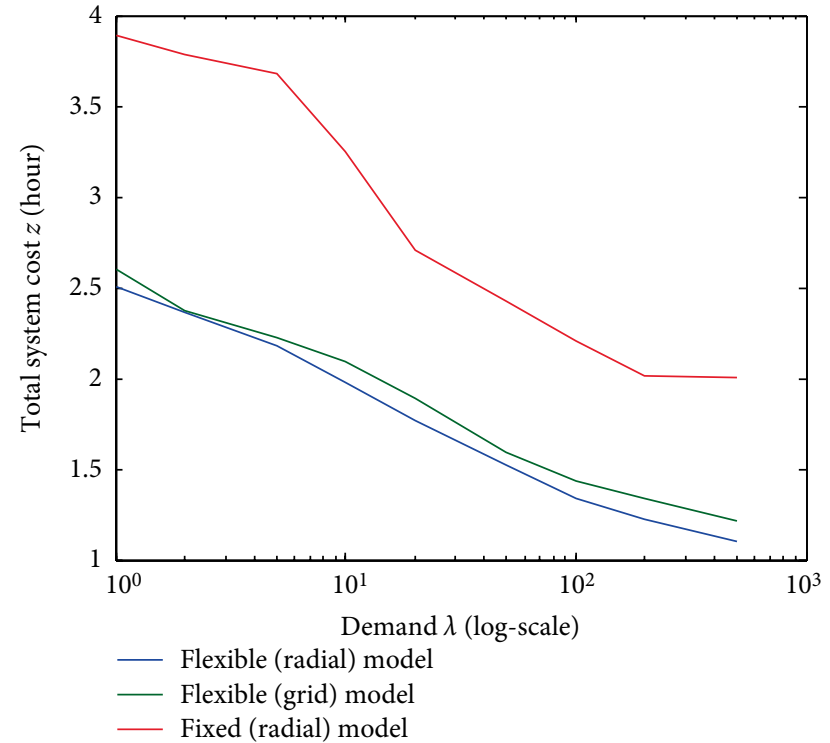

(b)

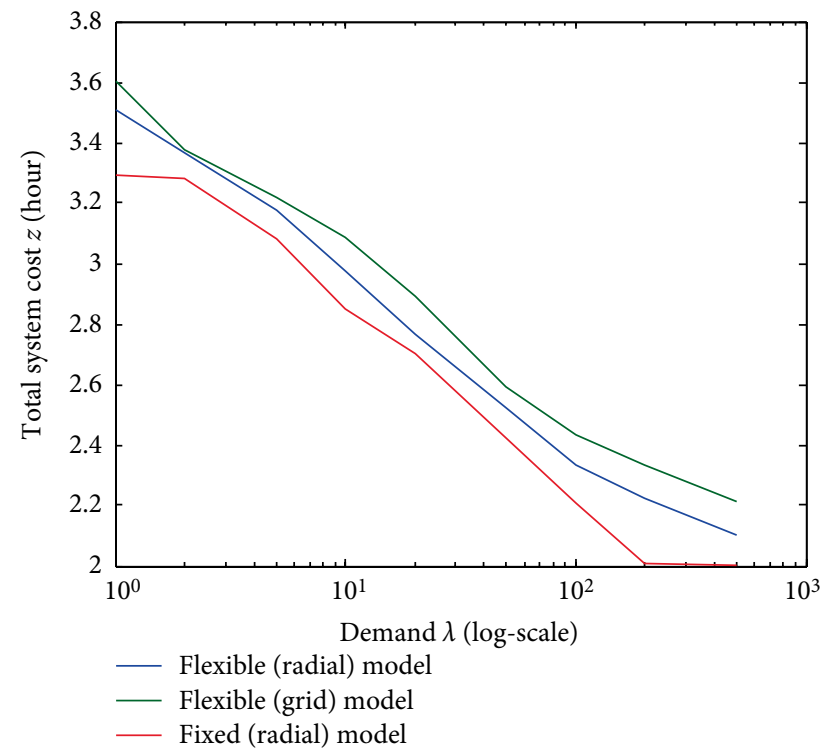

(d)

FIGURE 8: Minimized system cost versus demand levels with different input parameters. (a) $\tau=24 \mathrm{~s}$. (b) $v_{w}=1 \mathrm{~km} / \mathrm{h}$. (c) $v_{w}=7.5 \mathrm{~km} / \mathrm{h}$. (d) $\mu=80 \$ / \mathrm{h}$.

(iv) Also worth noting is that several factors, such as the city size, demand density level, value of time, in-vehicle travel time, are found to play an important role in the optimal alignment of the flexible transit system. However, the most important factors are in-vehicle travel time and demand density levels.

\section{Appendix}

\section{A. Derivations}

Please note that for an individual trip, it is likely to include some, not all of the travel time components. The design thought here is to take the average cost of the whole transit system into consideration rather than specific individual trips, which will exactly makes the optimal design problem too complicated. Now, we set out first to explore the expected number of transfers.

Result 1 . The expected number of transfers is given by

$$
\begin{aligned}
e_{T} & =0 \times \mathrm{P}(\operatorname{Tr}=0)+1 \times \mathrm{P}(\operatorname{Tr}=1) \\
& =1-\frac{1}{N_{r}}-\left(\frac{2}{\pi}-\frac{1}{2 N_{r}}\right) \frac{4 N_{c}^{2}-1}{3 N_{c}^{3}} .
\end{aligned}
$$

Derivation. To calculate the expected number of transfers, first we need to classify all trips into three categories: (1) the origin and destination of a trip are in the same influence area of a radial or circular line as depicted in Figure 9 (the solid dot 


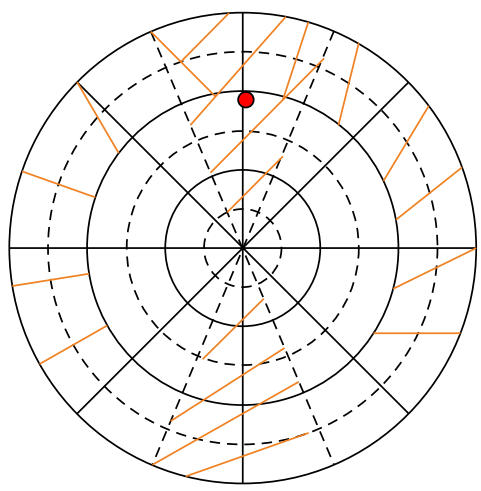

The origin

The same "tube" influence area of the origin

FIGURE 9: The same influence "tube" area of an origin.

represents the origin of a trip and the dash area represents the same "tube" influence area of the origin); (2) the destination is not in the same "tube" influence area of the origin but the angular between them is less than 2 (in radians); (3) the destination is not in the same "tube" influence area of the origin and the angular between them is more than 2 (in radians).

As regard to the first category, no transfer is needed. For the second category, passengers need to transfer at the transfer point located in the intersecting point of one radial "tube" and one circular "tube".

Hence, the expected number of transfers is

$$
\begin{aligned}
e_{T} & =0 \times \mathrm{P}(\operatorname{Tr}=0)+1 \times \mathrm{P}(\operatorname{Tr}=1) \\
& =1-\frac{1}{N_{r}}-\left(\frac{2}{\pi}-\frac{1}{2 N_{r}}\right) \frac{4 N_{c}^{2}-1}{3 N_{c}^{3}} .
\end{aligned}
$$

Let $\theta$ denote the angle between the origin and destination of a trip. Consideration shows that if $\theta>2$, passengers need to take two radial transit routes and a transfer is needed at the center. On the contrary, if $\theta<2$, passengers need to take one radial transit route, one circular route and transfer at the corresponding transfer point. Proofs can be found in Holroyd [23].

$$
\begin{aligned}
\mathrm{P}(\operatorname{Tr}=0)= & \sum_{i=1}^{N_{c}} \mathrm{P}_{o i} \mathrm{P}_{d i}=\sum_{i=1}^{N_{c}} \frac{\pi\left[i^{2} S_{c}^{2}-(i-1)^{2} S_{c}^{2}\right]}{\pi R^{2}} \\
& \times \frac{(1 / N)_{r} \pi R^{2}+\left(\left(4-\pi N_{r}\right) / 2 \pi\right) \pi \pi\left[i^{2} S_{c}^{2}-(i-1)^{2} S_{c}^{2}\right]}{\pi R^{2}} \\
= & \frac{1}{N_{r}}+\left(\frac{2}{\pi}-\frac{1}{2 N_{r}}\right) \frac{4 N_{c}^{2}-1}{3 N_{c}^{3}},
\end{aligned}
$$

where $\mathrm{P}_{o i}$ is the probability that the origin of a trip is in the $i$ th circular "tube" and $\mathrm{P}_{d i}$ is the probability that the destination of the trip is in the same "tube" influence area as the origin. According to the analysis above, a trip has one transfer at most, thus the probability of having one transfer is $[1-\mathrm{P}(\operatorname{Tr}=0)]$, which leads to $\mathrm{P}(\operatorname{Tr}=1)=1-\mathrm{P}(\operatorname{Tr}=0)=1-\left(1 / N_{r}\right)$ $-\left((2 / \pi)-\left(1 / 2 N_{r}\right)\right)\left(\left(4 N_{c}^{2}-1\right) / 3 N_{c}^{3}\right)$.
The expected number of transfers is

$$
\begin{aligned}
e_{T} & =0 \times \mathrm{P}(\operatorname{Tr}=0)+1 \times \mathrm{P}(\operatorname{Tr}=1) \\
& =1-\frac{1}{N_{r}}-\left(\frac{2}{\pi}-\frac{1}{2 N_{r}}\right) \frac{4 N_{c}^{2}-1}{3 N_{c}^{3}} .
\end{aligned}
$$

Result 2. Waiting time:

$$
\begin{aligned}
W & =\frac{H}{2}+\frac{H}{2} \mathrm{P}(\operatorname{Tr}=1) \\
& =\frac{H}{2}\left[2-\frac{1}{N_{r}}-\left(\frac{2}{\pi}-\frac{1}{2 N_{r}}\right) \frac{4 N_{c}^{2}-1}{3 N_{c}^{3}}\right] .
\end{aligned}
$$

Derivation. Due to the assumption of Poisson distribution, the average waiting time is half of the headway. Each passenger waits at the origin of a trip and at the transfer point if possible. Consequently, the waiting time can be calculated as

$$
\begin{aligned}
W & =\frac{H}{2}+\frac{H}{2} \mathrm{P}(T r=1) \\
& =\frac{H}{2}\left[2-\frac{1}{N_{r}}-\left(\frac{2}{\pi}-\frac{1}{2 N_{r}}\right) \frac{4 N_{c}^{2}-1}{3 N_{c}^{3}}\right] .
\end{aligned}
$$

Result 3. In-vehicle travel time (T):

$$
T=T_{c}+T_{r}=\frac{E_{c}}{v_{c}^{\prime}}+\frac{E_{r}}{v_{r}^{\prime}}=\frac{8 S_{c} \rho_{c} N_{r} N_{c}^{2}}{R^{2} v_{c}^{\prime}}+\frac{16 N_{r} N_{c} \rho_{r}}{\pi R v_{r}^{\prime}}
$$

Derivation. For circular routes, the average longitudinal distance to the transfer point in the $i$ th circular "tube" is $(1 / 4)(i-(1 / 2)) S_{c} \theta_{r}$. As to the lateral distance per passenger, it is difficult to derive a compact formulation using analytical methods. Following Chen and Nie [44], we denote $\rho_{c}$ as the ratio between the total travel distance and the longitudinal distance of a trip per vehicle. As we know, the longitudinal distance of a round trip per vehicle in the $i$ th "tube" is $4 \pi(i-(1 / 2)) S_{c}$, thus the total longitudinal distance per hour is $Q_{c}=\sum_{i=1}^{N_{c}} 4 \pi(i-(1 / 2)) S_{c}(1 / H)=\left(2 \pi N_{c} R / H\right)$. Then, the ratio $\rho_{c}$ can be expressed as $\rho_{c}=\left(Q_{c} / Q_{c^{\prime}}\right)=\left(\left(2 \pi R N_{c} / H\right)+\left(2 \lambda \pi R^{3} / 3 N_{c}\right)\right.$ $\left.+\left(R N_{r} / H N_{c}\right) /\left(2 \pi N_{c} R / H\right)\right)=1+\left(\lambda H R^{2} / 3 N_{c}^{2}\right)+\left(N_{r} / 2 \pi N_{c}^{2}\right)$.

Approximately, the in-vehicle travel distance per passenger on average can be calculated via scaling up the longitudinal distance by the ratio $\rho_{c}$. That leads to $E_{c}=\left(2 \sum_{i=1}^{N_{c}} 2 N_{r} \cdot 4 \pi(i-(1 / 2)) S_{c} \rho_{c} / \pi r^{2}\right)=\left(8 S_{c} \rho_{c} N_{r} N_{c}^{2} / R^{2}\right)$.

In a similar way, for radial routes, the longitudinal distance to the transfer point in the $i$ th circular "tube" is $(1 / 4) S_{c}$. The longitudinal distance for a round trip per vehicle of a radial "tube" is $4 \mathrm{R}$, thus the total longitudinal distance is $Q_{r}=\left(4 N_{r} R / H\right)$. The ratio $\rho_{c}$ is expressed as

$$
\begin{aligned}
\rho_{r} & =\frac{Q_{r}}{Q_{r^{\prime}}}=\frac{\left(4 R N_{r} / H\right)+2 \lambda \pi R^{2}+\left(5 \pi R N_{c} / 12 N_{r}\right)}{4 R N_{r} / H} \\
& =1+\frac{\lambda \pi R H}{2 N_{r}}+\frac{5 \pi N_{c} H}{48 N_{r}^{2}} .
\end{aligned}
$$


Therefore, the total travel distance per passenger in vehicles is

$$
E_{r}=2 \sum_{i=1}^{N_{c}} \frac{2 N_{r} \rho_{r} \cdot 4 R}{\pi R^{2}}=\frac{16 N_{r} N_{c} \rho_{r}}{\pi R} .
$$

In summary, the in-vehicle travel time per passenger is $T=T_{c}+T_{r}=\left(E_{c} / v_{c}^{\prime}\right)+\left(E_{r} / v_{r}^{\prime}\right)$.

Result 4. Vehicle distance

$$
Q=\frac{2 \pi R N_{c}}{H}+\frac{2 \lambda \pi R^{3}}{3 N_{c}}+\frac{R N_{r}}{H N_{c}}+\frac{4 R N_{r}}{H}+2 \lambda \pi R^{2}+\frac{5 \pi R N_{c}}{12 N_{r}} .
$$

Derivation. The total vehicle distance $Q$ is given by the product of the number of transit routes, the expected travel distance per round trip and the service frequencies (the inverse of the headway).

For the circular transit routes: the expected travel distance per round trip consists of three components: (1) the overcome longitudinal distance. For the $i$ th $\left(i=1,2, \ldots, N_{c}\right)$ from the center, the longitudinal distance per round trip is $4 \pi(i-(1 / 2)) S_{c} ;(2)$ the lateral movement to pick up and drop off passengers. The expected lateral distance per passenger is approximately $S_{c} / 3$ and the passenger trips generated during a round trip is $\lambda H \pi\left[i^{2} S_{c}^{2}-(i-1)^{2} S_{c}^{2}\right]$. Thus the expected lateral travel distance per round trip can be formulated as $2 \lambda H \pi\left[i^{2}-(i-1)^{2}\right] S_{c}^{3} / 3$ (considering both the pick-up and drop-off of each passenger); (3) the extra lateral movement due to compulsive stop at each transfer point. The expected lateral distance per transfer point is approximately $S_{c} / 4$. Hence, the total extra lateral distance is $\left(S_{c} / 4\right) \times 4 N_{r}$. To sum up, the expected vehicle distance per round trip is

$$
\begin{aligned}
Q_{c} & =\sum_{i=1}^{N_{c}}\left[4 \pi\left(i-\frac{1}{2}\right) \frac{R}{N_{c}}+\frac{2 \lambda H \pi\left[i^{2}-(i-1)^{2}\right] S_{c}^{3}}{3}+\frac{S_{c}}{4} \times 4 N_{r}\right] \times \frac{1}{H} \\
& =\frac{2 \pi R N_{c}}{H}+\frac{2 \lambda \pi R^{3}}{3 N_{c}}+\frac{R N_{r}}{H N_{c}} .
\end{aligned}
$$

The same reasoning applies for the radial flexible transit. For the radial transit routes, all the "tubes" are identical. The longitudinal distance is $4 R$ per round trip. The expected lateral distance in the $i$ th cordon is approximately $(i-(1 / 2))\left(S_{c} \theta_{r} / 3\right)$, and the passenger trips generated during a trip in the $i$ th cordon is $\lambda H \pi\left[i^{2} S_{c}^{2}-(i-1)^{2} S_{c}^{2}\right] / 2 N_{r}$. Besides, the extra lateral movement due to compulsive stops at designated transfer points is $(i-(1 / 2))\left(S_{c} \theta_{r} / 4\right)$. Thus, the expected vehicle distance per hour of operation is

$$
\begin{aligned}
Q_{r}= & \sum_{j=1}^{N_{r}}\left[4 R+2 \sum_{i=1}^{N_{c}}\left[\frac{2 \pi \lambda H\left[i^{2}-(i-1)^{2}\right] S_{c}^{2}}{2 N_{r}}\right.\right. \\
& \left.\left.+\left(i-\frac{1}{2}\right) \frac{S_{c} \theta_{r}}{3}+2 \times\left(i-\frac{1}{2}\right) \frac{S_{c} \theta_{r}}{4}\right]\right] \times \frac{1}{H} \\
= & \frac{2 \pi R N_{c}}{H}+\frac{2 \lambda \pi R^{3}}{3 N_{c}}+\frac{R N_{r}}{H N_{c}}+\frac{4 R N_{r}}{H}+2 \lambda \pi R^{2}+\frac{5 \pi R N_{c}}{12 N_{r}} .
\end{aligned}
$$

In total, the vehicle distance per hour of operation is expressed as $Q=Q_{c}+Q_{r}$.
Result 5. Fleet size:

$$
M=\frac{Q_{r}}{v_{r}^{\prime}}+2 \tau \lambda \pi R^{2}+\frac{Q_{c}}{v_{c}^{\prime}}+2 \tau \lambda \pi R^{2}
$$

Derivation. Via interpreting the definition of vehicle distance $Q$, we know that $Q$ is the unit distance travelled in one hour by all the vehicles. Thus the vehicle size can be easily derived as $M=\left(Q / v^{\prime}\right)$, where $v^{\prime}$ is the operating speed. For the circular transit routes, the time required to travel $Q_{c}$ includes: (1) overcome distance $Q_{c} / v$; (2) pick-up and drop-off time $2 \tau \lambda \pi R^{2}$. Thus, the vehicle size for the radial routes can be derived as $M_{c}=\left(Q_{c} / v_{c}^{\prime}\right)+2 \tau \lambda \pi R^{2}$. In the same way, the vehicle size for the radial routes can be derived as $M_{r}=\left(Q_{r} / v_{r}^{\prime}\right)+2 \tau \lambda \pi R^{2}$.

\section{Data Availability}

No data were used to support this study.

\section{Additional Points}

Future Work. In this paper, we assume the uniform distribution. One can assume other density patterns, for example, concentric demand or multi-pole demand patterns. This opens way for new research. It would also be intriguing to analyze if the objective function can be the total social benefit, taking the external cost into consideration although these further thoughts will undoubtedly add great complexity into the optimal design problem. Another line of work worthy to be done is to explore the hybrid structure of the flexible transit system proposed in this paper. For example, one can consider combining the hybrid structure of the one proposed by Daganzo [33] with it, providing double coverage in the central service area and only radial service in the periphery band. With the rapid development of technology and sharing economy, adding e-hailing service into the transit system calls for further investigation. Limitations. Apart from remarkable advantages, on the downside, a flexible transit system like the one proposed in this paper may impose significant in-vehicle travel time penalties on passengers. Happily, these penalties can be relieved by: (1) providing a nice and comfortable environment in the vehicles; (2) utilizing the in-vehicle time fully. As regards the second one, thanks to the rapid development of Internet and communication technology, passengers no longer need to waste their in-vehicle travel time. Rather, they can deal with their own business if they wish, which, to a large extent, reduces the disutility of the in-vehicle time. All of the above notwithstanding, we acknowledge that this kind of transit design proposed in this paper may be a bit divorced from reality. Actually, cities have two circular lines at most around the world. However, the work we do in this paper is not primarily to allocate its use in real cases. Instead, we choose this simple and compact form to obtain useful insights into the relation between the input parameters and the outcome of the optimal design topology. 


\section{Conflicts of Interest}

The authors declare that they have no conflicts of interest.

\section{Acknowledgments}

This paper was founded by National Natural Science Foundation of China (61803314).

\section{References}

[1] P. W. Chen and Y. M. Nie, "Analysis of an idealized system of demand adaptive paired-line hybrid transit," Transportation Research Part B: Methodological, vol. 102, pp. 38-54, 2017.

[2] P. Chen and Y. M. Nie, "Connecting e-hailing to mass transit platform: analysis of relative spatial position," Transportation Research Part C: Emerging Technologies, vol. 77, pp. 444-461, 2017.

[3] H. Badia, M. Estrada, and F. Robusté, "Competitive transit network design in cities with radial street patterns," Transportation Research Part B: Methodological, vol. 59, pp. 161-181, 2014.

[4] S. M. Nourbakhsh and Y. Ouyang, "A structured flexible transit system for low demand areas," Transportation Research Part B: Methodological, vol. 46, no. 1, pp. 204-206, 2012.

[5] A. Ceder and N. H. Wilson, "Bus network design," Transportation Research Part B: Methodological, vol. 20, no. 4, pp. 331-344, 1986.

[6] J. C. Rea, "Designing urban transit systems: an approach to the route-technology selection problem," Public Transportation and Passenger Characteristics, Highway Research Board, pp. 48-59, 1972.

[7] C. E. Mandl, "Evaluation and optimization of urban public transportation networks," European Journal of Operation Research, vol. 5, no. 6, pp. 396-404, 1980.

[8] M. H. Baaj and H. S. Mahmassani, "An AI-based approach for transit route system planning and design," Journal of Advanced Transportation, vol. 25, no. 2, pp. 187-209, 1991.

[9] M. H. Baaj and H. S. Mahmassani, "Hybrid route generation heuristic algorithm for the design of transit networks," Transportation Research Part C: Emerging Technologies, vol. 3, no. 1, pp. 31-50, 1995.

[10] F.Zhao, "Large-scale transit network optimization by minimizing user cost and transfers," Journal of Public Transportation, vol. 9, no. 2, pp. 107-129, 2006.

[11] F. Zhao and X. Zeng, "Optimization of transit route network, vehicle headways and timetables for large-scale transit networks," European Journal of Operation Research, vol. 186, no. 2, pp. 841-855, 2008.

[12] V. Guihaire and J.-K. Hao, "Transit network design and scheduling: a global review," Transportation Research Part A: Policy and Practice, vol. 42, no. 10, pp. 1251-1273, 2008.

[13] W. Szeto and Y. Wu, "A simultaneous bus route design and frequency setting problem for Tin Shui Wai, Hong Kong," European Journal of Operation Research, vol. 209, no. 2, pp. 141155, 2011.

[14] S. Wirasinghe and P. Seneviratne, "Rail line length in an urban transportation corridor," Transportation Science, vol. 20, no. 4, pp. 237-245, 1986.
[15] P. Jehiel, "Equilibrium on a traffic corridor with several congested modes," Transportation Science, vol. 27, no. 1, pp. 16-24, 1993.

[16] Z.-C. Li, W. H. Lam, and S. Wong, "Modeling intermodal equilibrium for bimodal transportation system design problems in a linear monocentric city," Transportation Research Part B: Methodological, vol. 46, no. 1, pp. 30-49, 2012.

[17] B. F. Byrne and V. R. Vuchic, "Public transportation line positions and headways for minimum cost," Traffic Flow Transportation, pp. 347-360, 1972.

[18] V. Hurdle, "Minimum cost locations for parallel public transit lines," Transportation Science, vol. 7, no. 4, pp. 340-350, 1973.

[19] B. F. Byrne, "Cost minimizing positions, lengths and headways for parallel public transit lines having different speeds," Transportation Research, vol. 10, no. 3, pp. 209-214, 1976.

[20] S. Wirasinghe, "Nearly optimal parameters for a rail/feeder-bus system on a rectangular grid," Transportation Research Part A: General, vol. 14, no. 1, pp. 33-40, 1980.

[21] S. K. Chang and P. M. Schonfeld, "Multiple period optimization of bus transit systems," Transportation Research Part B: Methodological, vol. 25, no. 6, pp. 453-478, 1991.

[22] S. K. Chang and P. M. Schonfeld, "Optimization models for comparing conventional and subscription bus feeder services," Transportation Science, vol. 25, no. 4, pp. 281-298, 1991.

[23] E. Holroyd, "The optimum bus service: a theoretical model for a large uniform urban area," in Proceedings of the Third International Symposium on the Theory of Traffic Flow, vol. 1, pp. 308-328, OECD 1967, NY, USA, 1967.

[24] S. Wirasinghe and N. S. Ghoneim, "Spacing of bus-stops for many to many travel demand," Transportation Science, vol. 15, no. 3, pp. 210-221, 1981.

[25] G. Kocur and C. Hendrickson, "Design of local bus service with demand equilibration," Transportation Science, vol. 16, no. 2, pp. 149-170, 1982.

[26] V. R. Vuchic and G. F. Newell, "Rapid transit interstation spacings for minimum travel time," Transportation Science, vol. 2, no. 4, pp. 303-339, 1968.

[27] B. F. Byrne, "Public transportation line positions and headways for minimum user and system cost in a radial case," Transportation Research, vol. 9, no. 2, pp. 97-102, 1975.

[28] S. C. Wirasinghe, V. F. Hurdle, and G. F. Newell, "Optimal parameters for a coordinated rail and bus transit system," Transportation Science, vol. 11, no. 4, pp. 359-374, 1977.

[29] L. N. Spasovic and P. M. Schonfeld, "Method for optimizing transit service coverage," Transportation Research Record, vol. 1402, pp. 28-39, 1993.

[30] H. Chen, W. Gu, M. J. Cassidy, and C. F. Daganzo, "Optimal transit service atop ring-radial and grid street networks: a continuum approximation design method and comparisons," Transportation Research Part B: Methodological, vol. 81, pp. 755-774, 2015.

[31] G. F. Newell, "Some issues relating to the optimal design of bus routes," Transportation Science, vol. 13, no. 1, pp. 20-35, 1979.

[32] E. M. Holroyd, “The optimum bus service: a theoretical model for a large uniform urban area," in Proceedings of the $3 \mathrm{rd}$ International Symposium on the Theory of Traffic Flow, L. C. Edie, R. Herman, and R. Rothery, Eds., Elsevier, NY, USA, 1965, Vehicular Traffic Science.

[33] C. F. Daganzo, "Structure of competitive transit networks," Transportation Research Part B: Methodological, vol. 44, no. 4, pp. 434-446, 2010. 
[34] M. Estrada, M. Roca-Riu, H. Badia, F. Robusté, and C. F. Daganzo, "Design and implementation of efficient transit networks: procedure, case study and validity test," Transportation Research Part A: Policy and Practice, vol. 45, no. 9, pp. 935-950, 2011.

[35] S. Saidi, S. Wirasinghe, and L. Kattan, "Long-term planning for ring-radial urban rail transit networks," Transportation Research Part B: Methodological, vol. 86, pp. 128-146, 2016.

[36] G. L. Thompson, "Planning considerations for alternative transit route structures," Journal of the American Institute of Planners, vol. 43, no. 2, pp. 158-168, 1977.

[37] S. R. Jara-Díaz and A. Gschwender, "From the single line model to the spatial structure of transit services: corridors or direct?" Journal of Transport Economics and Policy, vol. 37, no. 2, pp. 261-277, 2003.

[38] G. L. Thompson and T. G. Matoff, "Keeping up with Joneses: planning for transit in decentralizing regions," Journal of the American Planning Association, vol. 69, no. 3, pp. 296-312, 2003.

[39] J. R. Brown and G. L. Thompson, "Examining the Influence of multi-destination service orientation on transit service productivity: a multivariate analysis," Transportation, vol. 35, no. 2, pp. 237-252, 2008.

[40] P. Mees, A Very Public Solution: Transport in the Dispersed City, Melbourne University Press, Melbourne, 2000.

[41] P. Schimek, "Understanding differences in public transit: comparison of Boston and Toronto," Transportation Research Record: Journal of the Transportation Research Board, vol. 1604, no. 1, pp. 9-17, 1997.

[42] J. R. Brown and G. L. Thompson, "Should transit serve the CBD or a diverse array of destinations? a case study comparison of two transit systems," Journal of Public Transportation, vol. 15, no. 1, pp. 1-18, 2012.

[43] W. Fan, Y. Mei, and W. H. Gu, "Optimal design of intersecting bimodal transit networks in a grid city," Transportation Research Part B: Methodological, vol. 111, pp. 203-226, 2018.

[44] P. Chen and Y. M. Nie, "Optimal design of demand adaptive paired-line hybrid transit: case of radial route structure," Transportation Research Part E: Logistics and Transportation Review, vol. 110, pp. 71-89, 2018. 


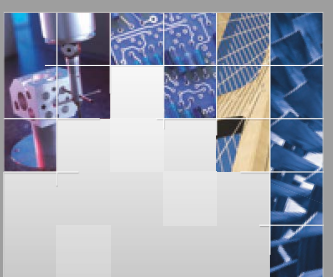

\section{Enfincering}
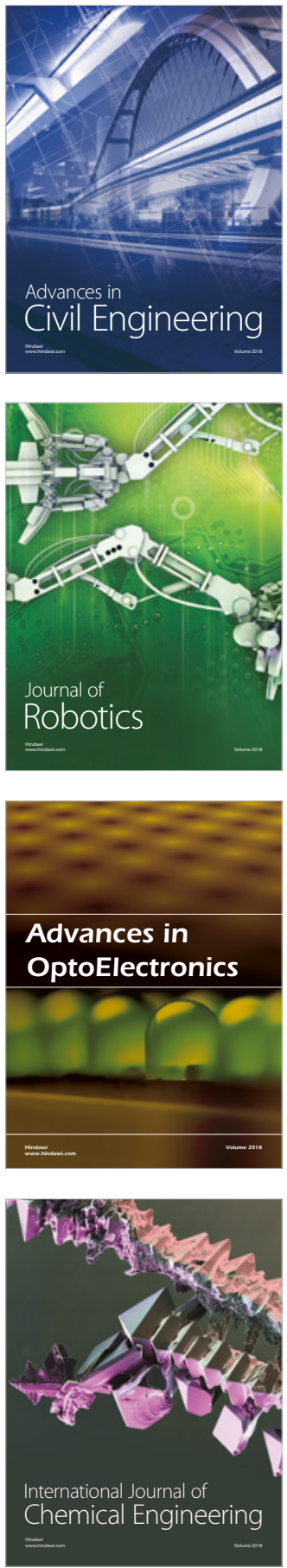

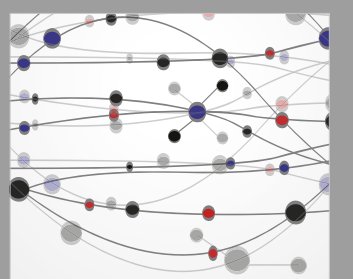

\section{Rotating \\ Machinery}

The Scientific World Journal

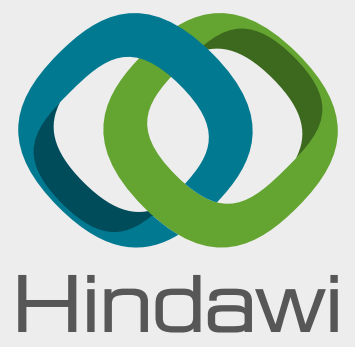

Submit your manuscripts at

www.hindawi.com
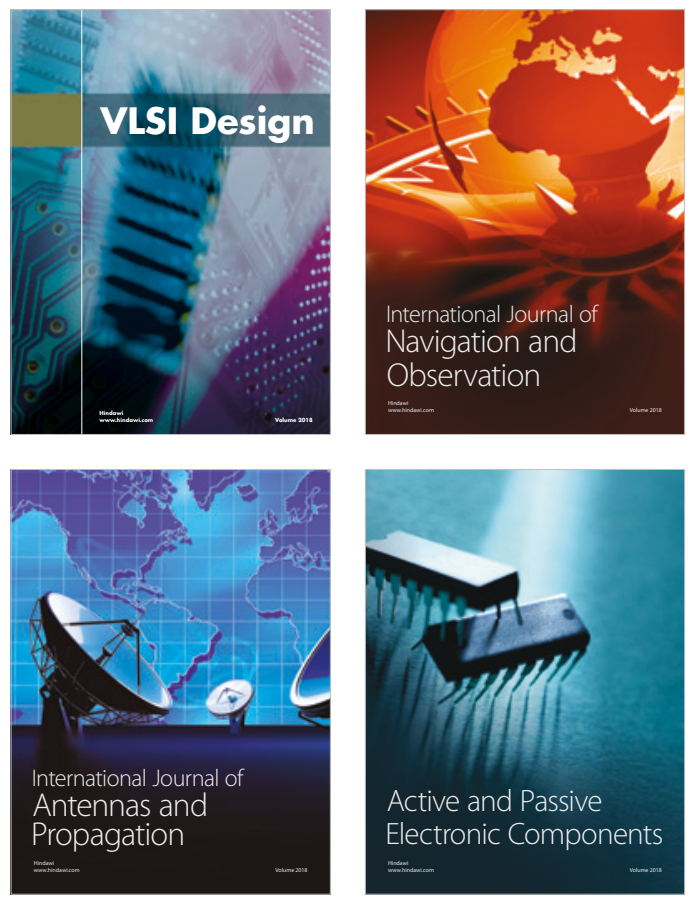
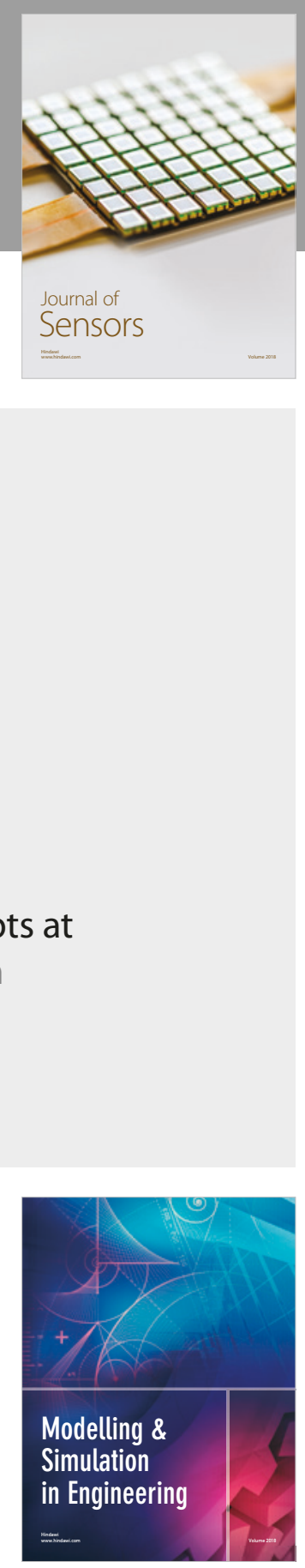

\section{Advances \\ Multimedia}
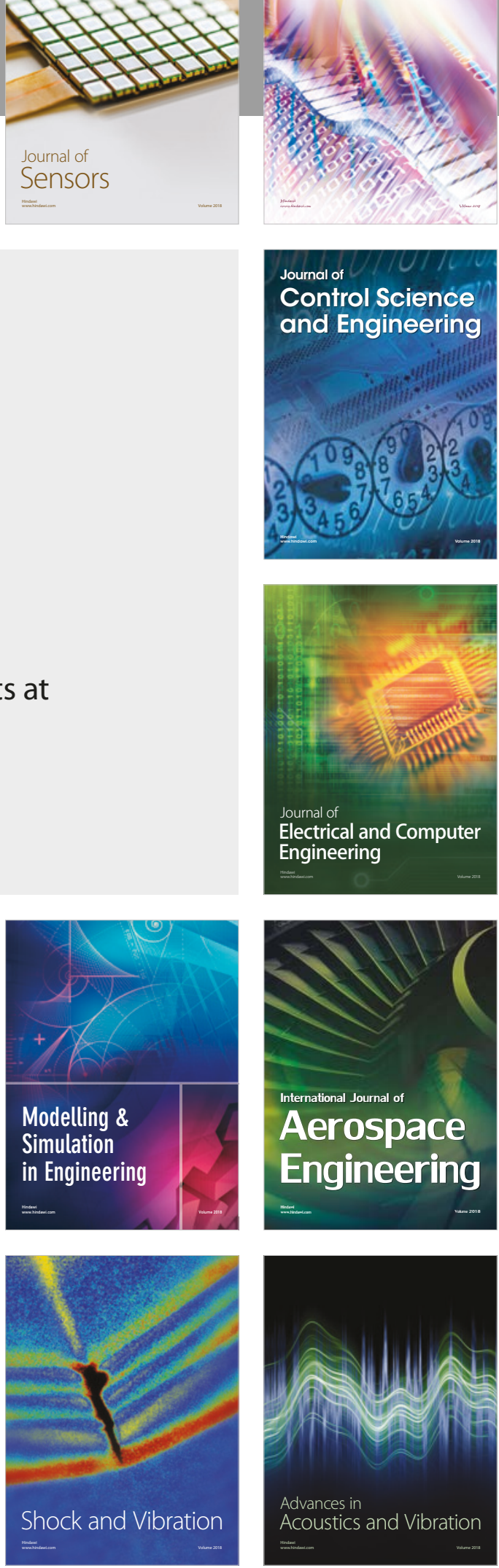